\title{
Two Solar Observation Programmes
}

Marie J. Marthes, Jean M. Malherbe, and J. Vial

Observatoire de Paris, 5, pl. Jules Janssen, F-92195 Meudon Principal Cedex, France

The Solar Section of the Societé Astronomique de France has organized various programmes designed to enable amateurs to carry out high-quality and scientifically useful observations. Two particular programmed will be described.

\section{The Study of the Proper Motions of Sunspots}

This was started in 1974, and was aimed at all potential observers. It was carried out under professional guidance and was designed to determine the speed of rotation of major spots and their proper motion in heliographic latitude and longitude. Further statistical study aimed at examining the data as a function of the period of the cycle (the latitude effect); relative positions (interactions between groups); departures from average motion (mean differential rotation). Sunspots are tracers of the magnetic field and there appearance is linked with activity in deep layers of the Sun that are not directly accessible to observation. The proper motion of spots in their first days of life is determined by these inner layers, so these studies are relevant to understanding convection within the Sun.

Observing was in white light and the programme covered the period from 1973 to 1982 (Cycle 21). A minimum of standardization was required, observers being able to observe whenever they could and would, with their own instruments and techniques. They were encouraged to continue to derive Wolf numbers themselves and, finally, were only given instructions and advice when they felt they wanted them. In return for their efforts they learnt to determine the position of the solar axis, to follow solar rotation from the motion of sunspots, to calculate heliographic coordinates, and possibly to take part in the analysis of the data received.

This programme, covering the rise to maximum of Cycle 21 and the subsequent decline was a great success. More than 40000 positional determinations were received, exceeding all expectations, and causing some problems for the Section in handling all the information. Although theoretically finishing with Cycle 21, this programme may be revived as spots become more numerous (the maximum of Cycle 22 is predicted to be $1990-1$ ). 


\section{Solar Prominence Programme}

The programme is due to start in 1988 and involves systematic $H-\alpha$ observations of prominences at the solar limb, with particular reference to two types of instability: those associated with quiescent prominences - sudden disappearances (which remain poorly understood), and bridges by which material passes from one prominence to another - and those events not linked to existing prominences, such as surges, ejection of material and eruptions. In the latter case the phenomena are associated with active centres, plages, and spots.

Three types of study are proposed: morphology, kinematics - for example, the various parameters of the apparent motion against the plane of the sky can be calculated and compared with typical values of velocities, time, and acceleration and finally, comparison of the phenomena as seen against the sky with similar features seen against the solar disk, and thus trying to understand the three-dimensional structure.

Analysis will be by a combined team of professionals and amateurs. Far from being a hindrance, the great variety of structures that can be observed are an added incentive to obtain as many observations as possible so that they may be classified and brought into an overall scheme. The wide variety of equipment used and the geographical distribution of amateurs will be of great value in carrying out this work. 